\title{
Longitudinal distribution of initial energy density and directed flow of charged particles in relativistic heavy-ion collisions
}

\author{
Ze-Fang Jiang $\odot,{ }^{1,2, *}$ Shanshan Cao $\odot,{ }^{3, \dagger}$ Xiang-Yu Wu, ${ }^{1}$ C. B. Yang $\odot,{ }^{1}$ and Ben-Wei Zhang ${ }^{1}$ \\ ${ }^{1}$ Institute of Particle Physics and Key Laboratory of Quark and Lepton Physics (MOE), \\ Central China Normal University, Wuhan, Hubei, 430079, China \\ ${ }^{2}$ Department of Physics and Electronic-Information Engineering, Hubei Engineering University, Xiaogan, Hubei, 432000, China \\ ${ }^{3}$ Institute of Frontier and Interdisciplinary Science, Shandong University, Qingdao, Shandong 266237, China
}

(Received 13 December 2021; accepted 18 February 2022; published 2 March 2022)

\begin{abstract}
We study the origin of the directed flow of charged particles produced in relativistic heavy-ion collisions. Three different initial conditions, Bozèk-Wyskiel, CCNU, and Shen-Alzhrani, of energy density distributions are coupled to the $(3+1)$-dimensional viscous hydrodynamic model CLVisc, and their effects on the development of the anisotropic medium geometry, pressure gradient, and radial flow are systematically compared. By comparison to experimental data at both the Relativistic Heavy-Ion Collider and the Large Hadron Collider, we find that the directed flow provides a unique constraint on the tilt of the initial medium profile in the plane spanned by the impact parameter and space-time rapidity. Within midrapidity, the counterclockwise tilt is shown to be a crucial source of the positive-negative force by the pressure gradient along the impact parameter $(x)$ direction at backward-forward rapidity, which drives a negative slope of the $x$ component of the medium flow velocity with respect to rapidity, and in the end the same feature of the charged particle directed flow.
\end{abstract}

DOI: 10.1103/PhysRevC.105.034901

\section{INTRODUCTION}

Heavy-ion collisions at the BNL Relativistic Heavy-Ion Collider (RHIC) and the CERN Large Hadron Collider (LHC) suggest that a hot and dense nuclear matter, known as quarkgluon plasma (QGP), is formed in the reaction region. The strong collective flow, such as the elliptic flow $\left(v_{2}\right)$, of the observed hadrons in various collision systems [1-3] is one of the most important signatures of the strongly interacting nature of the QGP. It has been successfully described by relativistic hydrodynamic models [4-24], and the specific shear viscosity extracted from the model-to-data comparison is shown to be small $[25,26]$.

The first-order Fourier coefficient of the azimuthal distribution of hadrons, also known as the rapidity-odd directed flow $\left(v_{1}\right)[27,28]$, is among the earliest observables for studying the collectivity in nuclear collisions at different energies [29-31]. Since the commencement of relativistic heavy-ion collisions, it has been widely studied in RHIC and LHC experiments as well [32-37]. Model calculations suggest that directed flow is initiated during the passage time of the two colliding nuclei, whose typical timescale is $2 R / \gamma$ with $R$ and $\gamma$ being

\footnotetext{
*jiangzf@mails.ccnu.edu.cn

†shanshan.cao@sdu.edu.cn
}

Published by the American Physical Society under the terms of the Creative Commons Attribution 4.0 International license. Further distribution of this work must maintain attribution to the author(s) and the published article's title, journal citation, and DOI. Funded by $S C O A P^{3}$. the nuclear radius and Lorentz contraction factor respectively $[6,29,38]$. This could be earlier than the development of elliptic flow. Therefore, $v_{1}$ has been considered a sensitive probe of the fireball size and nucleon flow at the initial stage $[4,27,38$ 42]. There are various sources contributing to the directed flow. It has been proposed that the size and sign of $v_{1}$ could depend on the deformation of the initial medium geometry, the baryon current, the equation of state of nuclear matter, and hadronic rescatterings [40,43-49], although their quantitative relative contributions are still open questions.

To investigate how the initial geometric asymmetry is transformed to the final hadron $v_{1}$, various initialization methods have been developed and coupled to hydrodynamic model calculations. Among different parametrizations of the longitudinal structure of the initial fireball, one of the most frequently applied approaches is the Bozek-Wyskiel parametrization proposed in Ref. [44]. It takes into account the asymmetry along the impact parameter direction $(x)$ at different spacetime rapidity $\left(\eta_{s}\right)$, which generates a counterclockwise tilt of the initial fireball in the $x-\eta_{s}$ plane. This initialization method has been successful in understanding the directed flow of not only the soft hadron but also the heavy flavor mesons [40,48-50] after coupling the heavy quark evolution model to the titled QGP medium. Following the idea of Bozèk-Wyskiel, we developed an alternative initialization ansatz (Central China Normal University - CCNU parametrization) of the longitudinal distribution of the nuclear matter in an earlier study [51]. It grasps the key feature of the tilted medium geometry and is able to describe the charged particle $v_{1}$ at RHIC and LHC. Recently, an additional collision geometry-based three-dimensional (3D) initial 
condition (Shen-Alzhrani parametrization) is developed in Refs. [46,47], which incorporates the Bjorken flow in the longitudinal direction (same as the Bozèk-Wyskiel and CCNU parametrizations), ensures the conservation of local energy and momentum, and provides a satisfactory description of the $v_{1}$ of $\pi^{+}$at RHIC. Therefore, it is of great interest to conduct a detailed comparison between these different initialization approaches within a uniform QGP evolution framework and identify the main features of the initial geometry that lead to the final state hadron $v_{1}$ we observe.

In this work, the above-mentioned systematical comparison between the three initial conditions-Bozèk-Wyskiel, CCNU, and Shen-Alzhrani-is performed using the $3+$ $1)$-D viscous hydrodynamic model CLVisc [52-54]. We investigate the correlation between the longitudinal structure of the initial fireball and the directed flow of the final-state charged particles. Our calculation indicates the counterclockwise tilt of the initial energy density profile yields an increasingdecreasing average pressure gradient $-\left\langle\partial_{x} P\right\rangle$ from zero with respect to time at backward-forward space-time rapidity within $\left|\eta_{s}\right|<2$. This further leads to a negative slope of the average QGP flow velocity $\left\langle v_{x}\right\rangle$ with respect to $\eta_{s}$, and in the end the same behavior of $v_{1}$ vs $\eta$ for the final-state charged particles, which is consistent with the experimental observations in $\sqrt{s_{N N}}=200 \mathrm{GeV} \mathrm{Au}+\mathrm{Au}$ collisions at RHIC [55] and $\sqrt{s_{N N}}=2.76 \mathrm{TeV} \mathrm{Pb}+\mathrm{Pb}$ collisions at LHC [56].

This article is organized as follows. In Sec. II, we will discuss three different initialization methods of the rapiditydependent energy density distribution in heavy-ion collisions and their impacts on the pressure gradient and flow velocity during the hydrodynamic expansion of the QGP. In Sec. III, we will present the charged particle directed flow from our hydrodynamic calculation and investigate its dependence on the initial condition. In the end, we summarize and discuss future improvements in Sec. IV.

\section{THE MODEL FRAMEWORK}

\section{A. Parametrizations of the initial energy density profile}

For the purpose of investigating the dependence of the final-state directed flow on the initial-state geometry of nuclear matter, in this section, we construct three different initial energy density distributions based on pioneer studies [44,46,47,51,57]. Their impacts on the time evolution of the pressure gradient and flow velocity of the QGP will be then explored using a $(3+1)$-D hydrodynamic model.

The nucleus thickness function $T(x, y)$ from the WoodsSaxon distribution is

$$
T(x, y)=\int_{-\infty}^{\infty} d z \frac{n_{0}}{1+e^{\left(\sqrt{x^{2}+y^{2}+z^{2}}-R\right) / d}},
$$

where $n_{0}$ is the average nuclear density, $d$ is the diffusiveness parameter, $x, y, z$ are the space coordinates, and $R$ is the radius of the nuclear Fermi distribution, which depends on the nucleus species. The parameters used for $\mathrm{Au}$ and $\mathrm{Pb}$ in the present study are listed in Table I.

Consider two nuclei propagate along $\pm \hat{z}$ and collide with the impact parameter $\mathbf{b}$. Their thickness function may be
TABLE I. Parameters used in the Woods-Saxon distribution for $\mathrm{Au}$ and $\mathrm{Pb}$ nuclei [58].

\begin{tabular}{lcccc}
\hline \hline Nucleus & $A$ & $n_{0}\left[1 / \mathrm{fm}^{3}\right]$ & $R[\mathrm{fm}]$ & $d[\mathrm{fm}]$ \\
\hline $\mathrm{Au}$ & 197 & 0.17 & 6.38 & 0.546 \\
$\mathrm{~Pb}$ & 208 & 0.17 & 6.62 & 0.535 \\
\hline \hline
\end{tabular}

written as

$$
T_{+}\left(\mathbf{x}_{\mathrm{T}}\right)=T\left(\mathbf{x}_{\mathrm{T}}-\mathbf{b} / 2\right), \quad T_{-}\left(\mathbf{x}_{\mathrm{T}}\right)=T\left(\mathbf{x}_{\mathrm{T}}+\mathbf{b} / 2\right),
$$

respectively, where $\mathbf{x}_{\mathrm{T}}=(x, y)$ is the transverse plane coordinate. The density distributions of participant nucleons from the two nuclei are then

$$
\begin{aligned}
& T_{1}\left(\mathbf{x}_{\mathrm{T}}\right)=T_{+}\left(\mathbf{x}_{\mathrm{T}}\right)\left\{1-\left[1-\frac{\sigma_{N N} T_{-}\left(\mathbf{x}_{\mathrm{T}}\right)}{A}\right]^{A}\right\}, \\
& T_{2}\left(\mathbf{x}_{\mathrm{T}}\right)=T_{-}\left(\mathbf{x}_{\mathrm{T}}\right)\left\{1-\left[1-\frac{\sigma_{N N} T_{+}\left(\mathbf{x}_{\mathrm{T}}\right)}{A}\right]^{A}\right\},
\end{aligned}
$$

where $A$ is the mass number of the colliding nuclei and $\sigma_{N N}$ is the inelastic nucleon-nucleon scattering cross section. The collision centrality classes are determined by the impact parameter b [58].

The right- and left-moving wounded nucleons are expected to produce more particles at forward and backward rapidity, respectively. This effect can be obtained by introducing the rapidity-dependent deformation into the weight function $W_{N}$ of wounded nucleons.

\section{Case (A): Bozè k-Wyskiel parametrization}

Following the Bozèk-Wyskiel parametrization of the tilted initial condition [44,57], two piecewise functions are used to construct the wounded nucleon weight function $W_{N}$ as

$$
W_{N}\left(x, y, \eta_{s}\right)=2\left[T_{1}(x, y) f_{+}\left(\eta_{s}\right)+T_{2}(x, y) f_{-}\left(\eta_{s}\right)\right],
$$

with

$$
f_{+}\left(\eta_{s}\right)= \begin{cases}0, & \eta_{s}<-\eta_{m}, \\ \frac{\eta_{s}+\eta_{m}}{2 \eta_{m}}, & -\eta_{m} \leqslant \eta_{s} \leqslant \eta_{m}, \\ 1, & \eta_{s}>\eta_{m},\end{cases}
$$

and

$$
f_{-}\left(\eta_{s}\right)= \begin{cases}1, & \eta_{s}<-\eta_{m}, \\ \frac{-\eta_{s}+\eta_{m}}{2 \eta_{m}}, & -\eta_{m} \leqslant \eta_{s} \leqslant \eta_{m}, \\ 0, & \eta_{s}>\eta_{m},\end{cases}
$$

in which $\eta_{s}$ is the longitudinal space-time rapidity, $\eta_{m}$, defines the range of rapidity correlations and affects the relative contribution from forward and backward participating nucleons.

\section{Case (B): CCNU parametrization}

In our earlier study [51], a monotonic function was introduced to describe the imbalance between the forward and backward regions, resulting in a tilted initial condition. The 
TABLE II. Common parameters shared between different initial condition parametrizations $[53,58]$.

\begin{tabular}{lcc}
\hline \hline & $\mathrm{Au}+\mathrm{Au} \sqrt{s_{N N}}=200 \mathrm{GeV} \mathrm{Pb}+\mathrm{Pb} \sqrt{s_{N N}}=2.76 \mathrm{TeV}$ \\
\hline$\tau_{0}(\mathrm{fm})$ & 0.6 & 0.6 \\
$K\left(\mathrm{GeV} / \mathrm{fm}^{3}\right)$ & 35.5 & 103.0 \\
$\alpha$ & 0.05 & 0.05 \\
$\sigma_{\mathrm{NN}}(\mathrm{mb})$ & 42 & 64 \\
$\eta_{w}$ & 1.3 & 2.0 \\
$\sigma_{\eta}$ & 1.5 & 1.8 \\
\hline \hline
\end{tabular}

weight function of wounded nucleons $W_{N}$ is defined as

$$
\begin{aligned}
W_{N}\left(x, y, \eta_{s}\right)= & {\left[T_{1}(x, y)+T_{2}(x, y)\right] } \\
& +H_{t}\left[T_{1}(x, y)-T_{2}(x, y)\right] \tan \left(\frac{\eta_{s}}{\eta_{t}}\right),
\end{aligned}
$$

where the parameter $H_{t}$ reflects the overall strength of imbalance between particle emission in the forward and backward rapidities along the direction of the impact parameter, while $\tan \left(\eta_{s} / \eta_{t}\right)$ generates the deformation of the initial energy density distribution along the rapidity direction. A fixed parameter of $\eta_{t}=8.0$ is used for all the collision systems investigated in the present work.

The initial energy density $\varepsilon\left(x, y, \eta_{s}\right)$ for both cases (A) and (B) is then given by [53]

$$
\varepsilon\left(x, y, \eta_{s}\right)=K W\left(x, y, \eta_{s}\right) H\left(\eta_{s}\right),
$$

where $K$ is an overall normalization factor that will be determined by the particle yield in different collision systems, and $W\left(x, y, \eta_{s}\right)$ is the total weight function defined as

$$
W\left(x, y, \eta_{s}\right)=\frac{(1-\alpha) W_{\mathrm{N}}\left(x, y, \eta_{s}\right)+\alpha n_{\mathrm{BC}}(x, y)}{\left.\left[(1-\alpha) W_{\mathrm{N}}(0,0,0)+\alpha n_{\mathrm{BC}}(0,0)\right]\right|_{\mathbf{b}=0}} .
$$

Here, $\alpha$ is known as the collision hardness parameter and $n_{\mathrm{BC}}(x, y)$ is the number of binary (hard) collisions given by

$$
n_{\mathrm{BC}}(x, y)=\sigma_{\mathrm{NN}} T_{+}(x, y) T_{-}(x, y) .
$$

In Eq. (7), a function

$$
H\left(\eta_{s}\right)=\exp \left[-\frac{\left(\left|\eta_{s}\right|-\eta_{w}\right)^{2}}{2 \sigma_{\eta}^{2}} \theta\left(\left|\eta_{s}\right|-\eta_{w}\right)\right]
$$

is introduced in order to describe the plateau structure of the rapidity distribution of emitted hadrons, in which $\eta_{w}$ determines the width of the central rapidity plateau while $\sigma_{\eta}$ determines the width (speed) of the Gaussian decay outside the plateau region [53]. Model parameters $-K, \eta_{w}$, and $\sigma_{\eta}-$ will be summarized in Table II soon.

\section{Case (C): Shen-Alzhrani parametrization}

A third parametrization of the $\eta_{s}$-dependent initial condition was proposed in Refs. [46,47], which ensures the local energy-momentum conservation during converting the two colliding nuclei into the energy density profile of the produced nuclear medium. The local invariant mass $M(x, y)$ and the center-of-mass rapidity $y_{\text {c.m. }}$ are respectively defined as

$$
\begin{aligned}
M(x, y) & =m_{\mathrm{N}} \sqrt{T_{1}^{2}+T_{2}^{2}+2 T_{1} T_{2} \cosh \left(2 y_{\text {beam }}\right)}, \\
y_{\text {c.m. }}(x, y) & =\operatorname{arctanh}\left[\frac{T_{1}-T_{2}}{T_{1}+T_{2}} \tanh \left(y_{\text {beam }}\right)\right],
\end{aligned}
$$

where $y_{\text {beam }}=\operatorname{arccosh}\left(\sqrt{s_{\mathrm{NN}}} / 2 m_{\mathrm{N}}\right)$ is the rapidity of each nucleon inside the colliding nuclei and $m_{\mathrm{N}}$ is the nucleon mass.

The local energy density profile is then modeled as [46]

$$
\begin{aligned}
\varepsilon\left(x, y, \eta_{s} ; y_{\text {c.m. }}\right)= & K \mathcal{N}_{e}(x, y) \\
\times & \exp \left[-\frac{\left(\left|\eta_{s}-\left(y_{\text {c.m. }}-y_{L}\right)\right|-\eta_{w}\right)^{2}}{2 \sigma_{\eta}^{2}}\right. \\
& \left.\times \theta\left(\left|\eta_{s}-\left(y_{\text {c.m. }}-y_{L}\right)\right|-\eta_{w}\right)\right],
\end{aligned}
$$

in which $K$ is the overall normalization factor and $y_{L}=f y_{\text {c.m. }}$. with $f \in[0,1]$. Here, the transverse density distribution $\mathcal{N}_{e}$ is determined by the local invariant mass $M(x, y)$ as

$$
\begin{aligned}
\mathcal{N}_{e}(x, y) & =\frac{M(x, y)}{M(0,0)\left[2 \sinh \left(\eta_{w}\right)+\sqrt{\frac{\pi}{2}} \sigma_{\eta} e^{\sigma_{\eta}^{2} / 2} C_{\eta}\right]}, \\
C_{\eta} & =e^{\eta_{w}} \operatorname{erfc}\left(-\sqrt{\frac{1}{2}} \sigma_{\eta}\right)+e^{-\eta_{w}} \operatorname{erfc}\left(\sqrt{\frac{1}{2}} \sigma_{\eta}\right),
\end{aligned}
$$

where $\operatorname{erfc}(x)$ is the complementary error function.

In Table II, we summarize the common parameters that are shared between our three model setups, cases (A), (B), and $(\mathrm{C})$, including the initial time of the hydrodynamic evolution $\left(\tau_{0}\right)$, overall normalization factor $(K)$, collision hardness parameter $(\alpha)$ [used in cases (A) and (B)], nucleon-nucleon inelastic cross section $\left(\sigma_{N N}\right)$, rapidity plateau width $\left(\eta_{w}\right)$, and the width of the Gaussian decay $\left(\sigma_{\eta}\right)$. They are tuned to provide a reasonable description of the charged hadron pseudorapidity distributions in the most central collisions [53], as will be shown in Figs. 7 and 8. Note that in order to compare the three parametrizations above in the same hydrodynamic evolution framework (CLVisc in this work), the parameters tuned here could be different from the original Bozèk-Wyskiel work for case (A) and Shen-Alzhrani work for case (C). The parameter designed for each specific model $-\eta_{m}$ for case (A), $H_{t}$ for case (B), and $f$ for case (C) - will be discussed later when we compare its corresponding model to the charged particle $v_{1}$ data.

The initial fluid velocity at $\tau_{0}$ is assumed to follow the Bjorken approximation in this work as $v_{x}=v_{y}=0$ and $v_{z}=$ $z / t$, where the initial transverse flow and the asymmetric distribution of $v_{z}$ along the $x$ direction are ignored. More sophisticated initial velocity profiles will be investigated in an upcoming study.

\section{B. Initial energy density, eccentricity, and pressure gradient}

With the parametrizations above, we first compare the energy density profile between different setups. In Fig. 1, we present the initial energy density at $\tau_{0}=0.6 \mathrm{fm}$ on the $\eta_{s}-x$ plane for $5-40 \% \mathrm{Au}+\mathrm{Au}$ collisions at $\sqrt{s_{N N}}=200 \mathrm{GeV}$. 

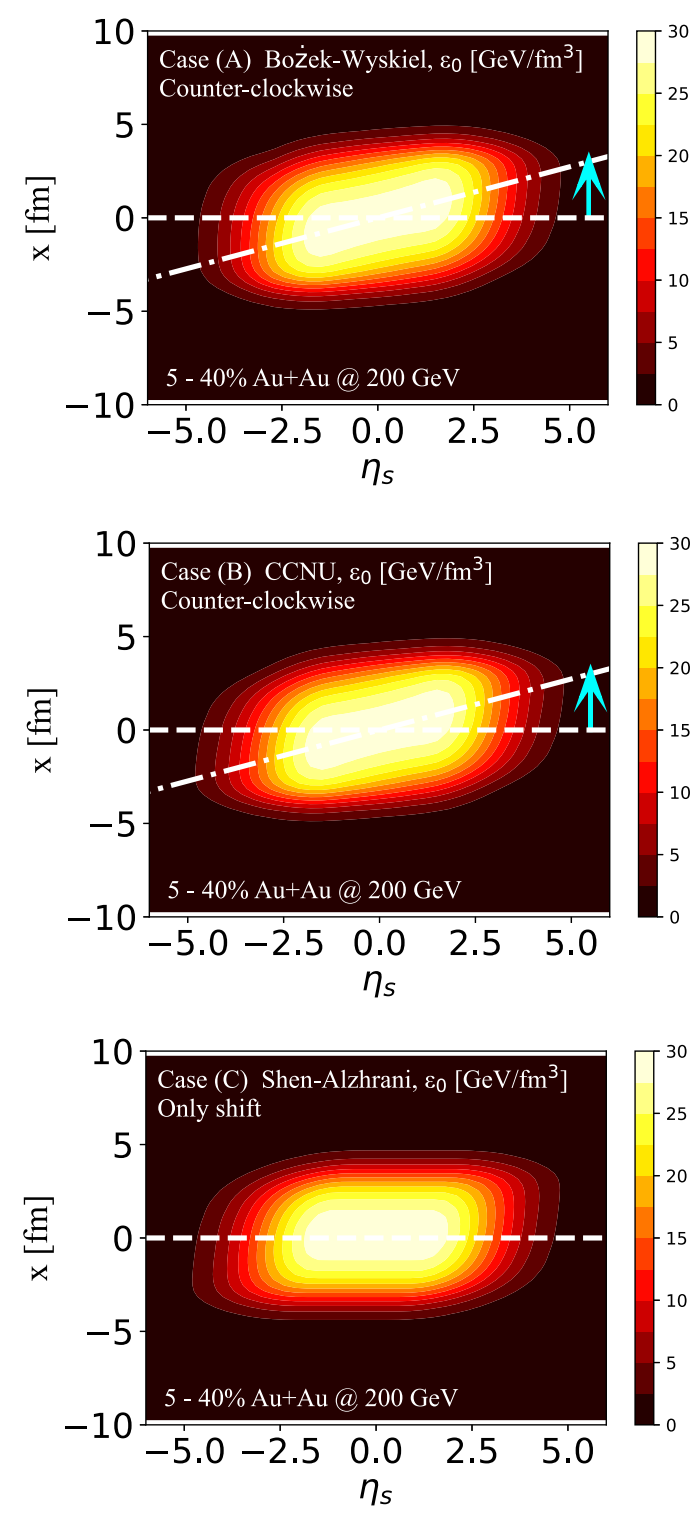

FIG. 1. The initial energy density on the $\eta_{s}-x$ plane at $\tau_{0}=0.6 \mathrm{fm}$ in $5-40 \%(b=6.7 \mathrm{fm}) \mathrm{Au}+\mathrm{Au}$ collisions at $\sqrt{s_{N N}}=200 \mathrm{GeV}$. From top to bottom panel, we present case (A) Bozek-Wyskiel parametrization with $\eta_{m}=2.8$, case (B) CCNU parametrization with $H_{t}=2.9$, and case (C) Shen-Alzhrani parametrization with $f=$ 0.15 . The arrows (aqua color) sketch the counterclockwise tilted initial condition with respect to the $x=0$ axis in the $\eta_{s}-x$ plane.

Three different parmetrizations of the initial energy distribution are compared. In order to describe the directed flow of charged particles later, the model parameter $\eta_{m}=2.8$ is taken for the Bozèk-Wyskiel parametrization (top panel) and $H_{t}=2.9$ for the CCNU parametrization (middle panel), while $f=0.15$ is taken from Ref. [47] for Shen-Alzhrani parametrization (bottom panel). From Fig. 1, we observe that parametrizations in our cases (A) and (B) generate similar initial energy density profiles: The distribution is not only shifted in the forward (backward) rapidity direction for the positive (negative) $x$ region, it is also tilted counterclockwise relative to $x=0$ in the $\eta_{s}-x$ plane (following the arrow direction in

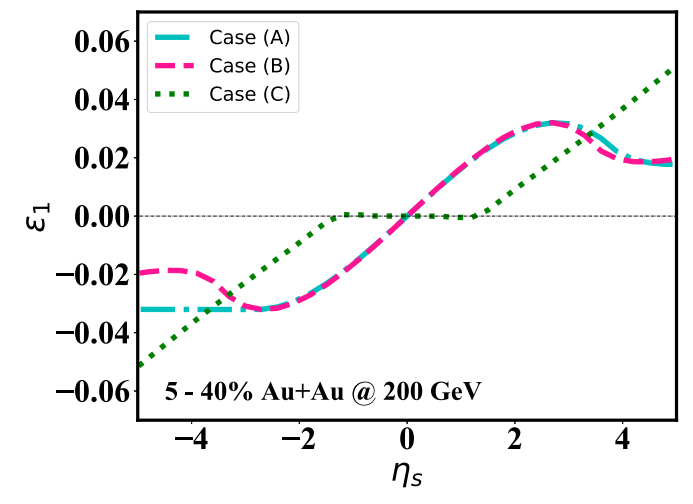

FIG. 2. The first-order eccentricity coefficient $\varepsilon_{1}\left(\eta_{s}\right)$ at $\tau_{0}=$ $0.6 \mathrm{fm}$ for $5-40 \% \mathrm{Au}+\mathrm{Au}$ collisions at $\sqrt{s_{N N}}=200 \mathrm{GeV}$, compared between three different model setups.

the figure). On the other hand, the distribution from case (C) only appears shifted horizontally in the rapidity direction. We note that our case (C) here is similar to the Hirano-Tsuda parametrization $[59,60]$ as illustrated in Ref. [44].

In order to quantify the asymmetry of the initial energy density distribution with respect to the $y-z$ plane from different models, we present their corresponding first-order eccentricity coefficient $\varepsilon_{1}$ in Fig. 2 as a function of the spacetime rapidity. The first-order eccentricity vector is defined as $[15,46]$

$$
\overrightarrow{\mathcal{E}}_{1} \equiv \varepsilon_{1}\left(\eta_{s}\right) e^{i \Psi_{1}\left(\eta_{s}\right)}=-\frac{\int d^{2} r \widetilde{r}^{3} e^{i \tilde{\phi}} \varepsilon\left(r, \phi, \eta_{s}\right)}{\int d^{2} r \widetilde{r}^{3} \varepsilon\left(r, \phi, \eta_{s}\right)},
$$

in which the angular distribution is evaluated with respect to the center-of-mass $\left(x_{0}\left(\eta_{s}\right), y_{0}\left(\eta_{s}\right)\right)$ of each rapidity slice given by

$$
\begin{aligned}
& x_{0}\left(\eta_{s}\right)=\frac{\int d^{2} r x \varepsilon\left(r, \phi, \eta_{s}\right)}{\int d^{2} r \varepsilon\left(r, \phi, \eta_{s}\right)}, \\
& y_{0}\left(\eta_{s}\right)=\frac{\int d^{2} r y \varepsilon\left(r, \phi, \eta_{s}\right)}{\int d^{2} r \varepsilon\left(r, \phi, \eta_{s}\right)} .
\end{aligned}
$$

The transverse radius and the azimuthal angle are then defined as $\widetilde{r}\left(x, y, \eta_{s}\right)=\sqrt{\left(x-x_{0}\right)^{2}+\left(y-y_{0}\right)^{2}}$ and $\widetilde{\phi}\left(x, y, \eta_{s}\right)=$ $\arctan \left[\left(y-y_{0}\right) /\left(x-x_{0}\right)\right]$ respectively. In the end, $\varepsilon_{1}$ in Eq. (16) gives the first-order eccentricity coefficient while $\Psi_{1}$ gives the corresponding participant plane angle. The $\eta_{s}$ dependence of this $\overrightarrow{\mathcal{E}}_{1}$ will contribute to explaining what kind of longitudinally deformed fireball is needed to produce the final hadron $v_{1}$.

In Fig. 2, one observes similar $\eta_{s}$ dependence of $\varepsilon_{1}$ between cases (A) and (B); they are odd functions of $\eta_{s}$ and positive (negative) in the $+\left(-\eta_{s}\right)$ regime. On the other hand, although $\varepsilon_{1}$ from case (C) is also an odd function of $\eta_{s}$, its value is much smaller than that from (A) and (B) within $\left|\eta_{s}\right|<2$. This will further affect the evolution profile of the nuclear medium in the subsequent hydrodynamic expansion.

In addition to the energy density distribution, we also present the initial pressure gradient $-\partial_{x} P$ in the transverse plane, which will directly drive the development of radial flow of nuclear matter. Our three model setups are compared in 

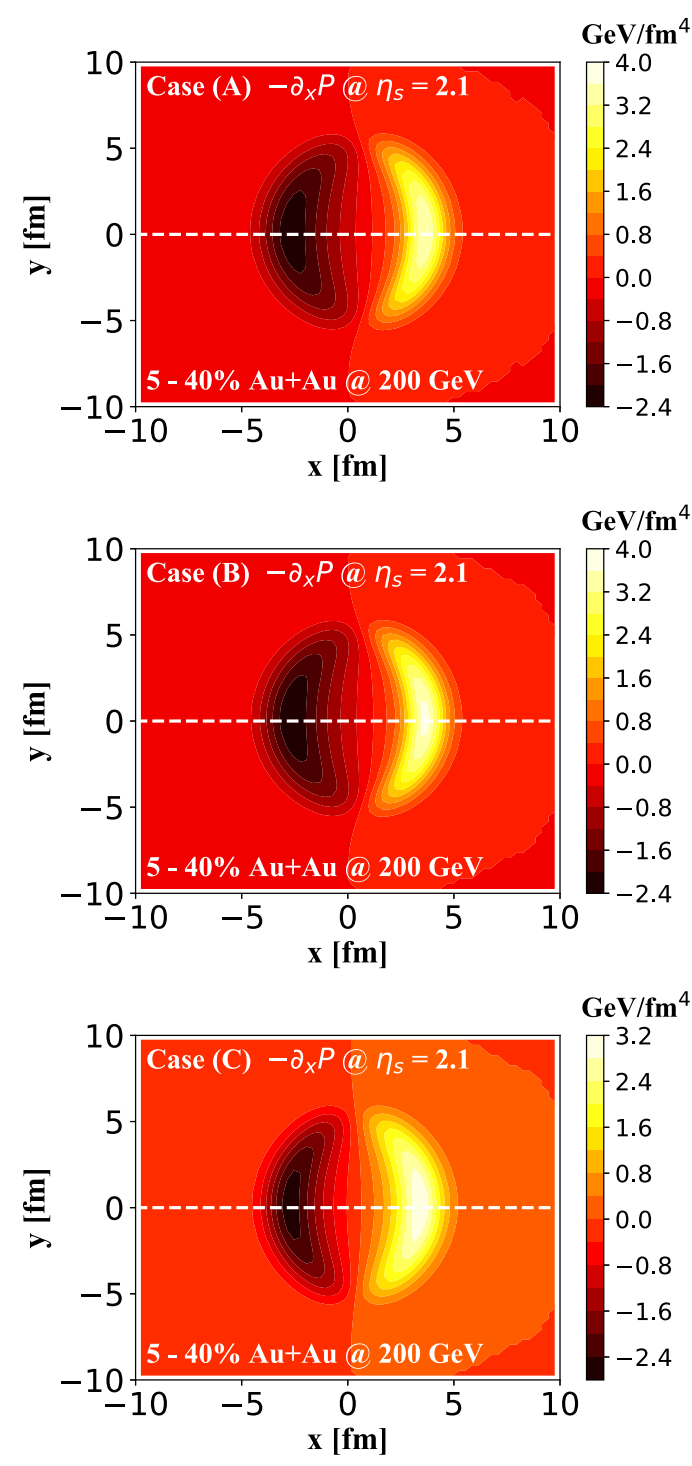

FIG. 3. The initial pressure gradient $-\partial_{x} P$ on the $x-y$ plane at $\tau_{0}=0.6 \mathrm{fm}$ and $\eta_{s}=2.1$ in $5-40 \%(b=6.7 \mathrm{fm}) \mathrm{Au}+\mathrm{Au}$ collisions at $\sqrt{s_{N N}}=200 \mathrm{GeV}$, compared between three different model setups.

Fig. 3 for the initial $-\partial_{x} P$ distribution in the $x-y$ plane at a given $\eta_{s}$, where the same parameter values of $\eta_{m}, H_{t}, f$ are used as for Fig. 1. One may clearly observe the positive (negative) value of $-\partial_{x} P$ in the $+(-x)$ direction that drives the outward expansion of the medium. In the top and middle panels, we see that at forward rapidity, the center (zero pressure) regions of these distributions are shifted toward $+x$ for cases (A) and (B) due to the counterclockwise tilt of the energy density distribution as previously discussed in Fig. 1. In contrast, such a shift is weaker in the bottom panel here for case (C) due to its different profile of energy density. Whether the average $x$ component of the final-state hadron momentum will be positive or negative at a given rapidity depends on the average value of this $-\partial_{x} P$ over the corresponding transverse plane and how it evolves with time. This will be discussed in detail later.

\section{Hydrodynamic evolution}

Starting with the initial energy density and flow velocity described above, we use the $(3+1)$-D viscous hydrodynamic model CLVisc [52-54,61,62] to simulate the subsequent evolution of the QGP medium in this work. The hydrodynamic equation reads [22,63-66]

$$
\partial_{\mu} T^{\mu v}=0,
$$

where $T^{\mu v}$ is the energy-momentum tensor defined as

$$
T^{\mu \nu}=\varepsilon u^{\mu} u^{\nu}-(P+\Pi) \Delta^{\mu \nu}+\pi^{\mu \nu} .
$$

It is composed of the local energy density $\varepsilon$, the fluid fourvelocity $u^{\mu}$, the pressure $P$, the shear stress tensor $\pi^{\mu \nu}$, and the bulk pressure $\Pi$. The projection tensor is given by $\Delta^{\mu \nu}=$ $g^{\mu \nu}-u^{\mu} u^{\nu}$, and the metric tensor $g^{\mu \nu}=\operatorname{diag}(1,-1,-1,-1)$ is used. The hydrodynamic equations are solved together with the lattice QCD equation of state (EoS) from the Wuppertal-Budapest group [67], and the shear-viscosity-toentropy-density ratio is set as $\eta_{v} / s=0.08$ ( $\eta_{v}$ for the shear viscosity) for all collision systems investigated in this work. However, we have ignored effects of bulk viscosity and net baryon density at this moment, which have been incorporated in the recent CLVisc development $[68,69]$ and will also be taken into account in our followup effort.

The isothermal freeze-out condition [53] is applied in the current calculation, where the freeze-out hypersurface is determined by a constant temperature value $T_{\text {frz }}=137 \mathrm{MeV}$. On this hypersurface, hadron spectra are evaluated based on the Cooper-Frye formalism [70]. Contributions from resonance decay have also been taken into account according to Ref. [53].

\section{Time evolution of average pressure gradient and flow velocity}

Hydrodynamic model describes how the asymmetry of the initial energy density distribution is transferred to the anisotropy of the final-state hadron momentum. In this subsection, using the hyrodynamic simulation, we investigate how the average pressure gradient $-\left\langle\partial_{x} P\right\rangle$ and flow velocity $\left\langle v_{x}\right\rangle$ develop with time at different $\eta_{s}$. This will help us understand the origin of directed flow and how it depends on the initial geometry of the nuclear matter.

As previously shown in Fig. 3, the deformation of the initial energy density breaks the symmetry of the pressure gradient along the $x$ direction. In Fig. 4, we study how the average pressure gradient over the transverse plane at a given space-time rapidity ( \pm 0.9 here) evolves with time. Similar to before, nuclear matter produced in 5-40\% Au + Au collisions at $\sqrt{s_{N N}}=200 \mathrm{GeV}$ is used. One finds that the time evolution of $-\left\langle\partial_{x} P\right\rangle$ is significantly affected by the initial condition of the medium. For cases (A) and (B) of our initial parametrization, $-\left\langle\partial_{x} P\right\rangle$ increases from zero with time at $\eta_{s}=-0.9$ while decreases at $\eta_{s}=0.9$, indicating an increasing overall force that accelerates the medium expansion toward the $+x$ direction at $\eta_{s}=-0.9$ while toward the $-x$ direction at $\eta_{s}=0.9$. Little difference is observed between the two parametrization methods in cases (A) and (B). In contrast, case (C) leads to a qualitatively opposite average pressure gradient, and therefore accelerating force, which slightly decreases from zero at at 

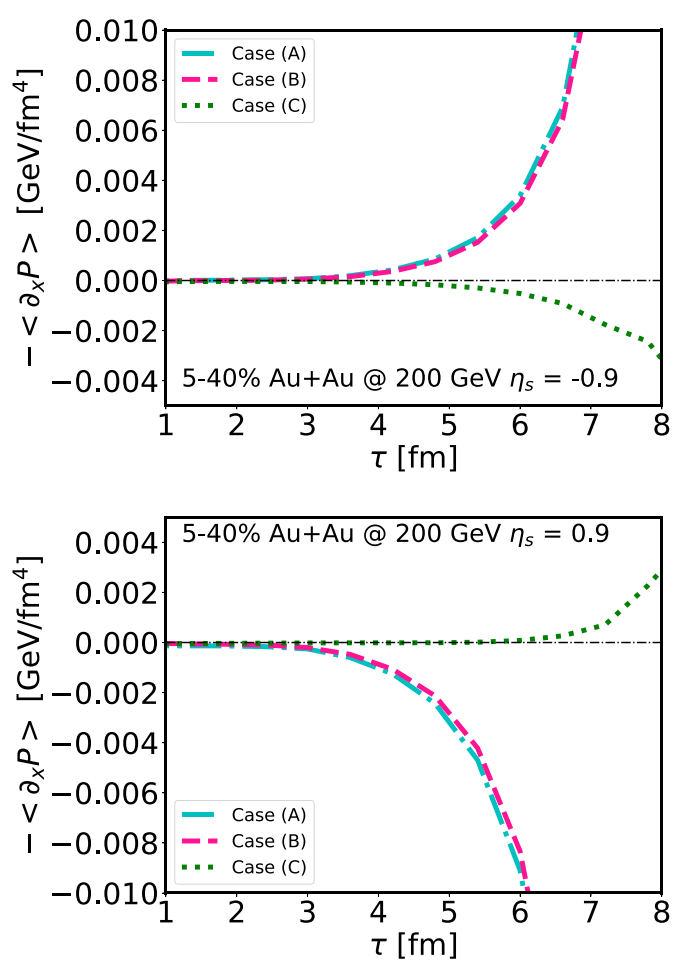

FIG. 4. Time evolution of the average pressure gradient in the $x$ direction at $\eta_{s}=-0.9$ (upper panel) and $\eta_{s}=0.9$ (lower panel) in $5-40 \% \mathrm{Au}+\mathrm{Au}$ collisions at $\sqrt{s_{N N}}=200 \mathrm{GeV}$, compared between three parametrization methods of the initial condition.

$\eta_{s}=-0.9$ but increases at $\eta_{s}=0.9$. Note that unlike higher order components of anisotropy, values of eccentricity $\varepsilon_{1}$ (in Fig. 2) and pressure gradient $-\left\langle\partial_{x} P\right\rangle$ are not necessarily positively correlated to each other. Due to the tilted deformation of medium profiles in cases (A) and (B), its expansion in space contributes to an overall force on the $+(-x)$ direction at backward (forward) rapidity, as intuitively illustrated in Ref. [44]. However, since the initial energy density given by case (C) is rather symmetric along the $\pm x$ direction at small $\left|\eta_{s}\right|$, different $-\left\langle\partial_{x} P\right\rangle$ is obtained.

The average pressure gradient can quantitatively or even qualitatively change as $\left|\eta_{s}\right|$ increases. Shown in Fig. 5 are the time evolution of $-\left\langle\partial_{x} P\right\rangle$ at $\eta_{s}=-2.1$ (upper panel) and 2.1 (lower panel). Compared to the smaller $\left|\eta_{s}\right|$ region (Fig. 4), one observes a larger magnitude of the pressure gradient for cases (A) and (B). Meanwhile, a sign flip is also found for case (C) due to its weaker asymmetric density distribution at smaller space-time rapidity but stronger asymmetric distribution at larger space-time rapidity along $\pm x$ direction, as can be seen in the bottom panel of Fig. 1.

A direct outcome of different pressure gradients is the different flow velocities in the corresponding direction. In Fig. 6, we study how the space-time rapidity dependence of the average flow velocity $\left\langle v_{x}\right\rangle$ develops with time. The average flow velocity at a given time and rapidity can be evaluated as $[16,44]$

$$
\left\langle v_{x}\left(\eta_{s}\right)\right\rangle=\frac{\int d^{2} r v_{x} \gamma \varepsilon\left(r, \phi, \eta_{s}\right)}{\int d^{2} r \gamma \varepsilon\left(r, \phi, \eta_{s}\right)}
$$
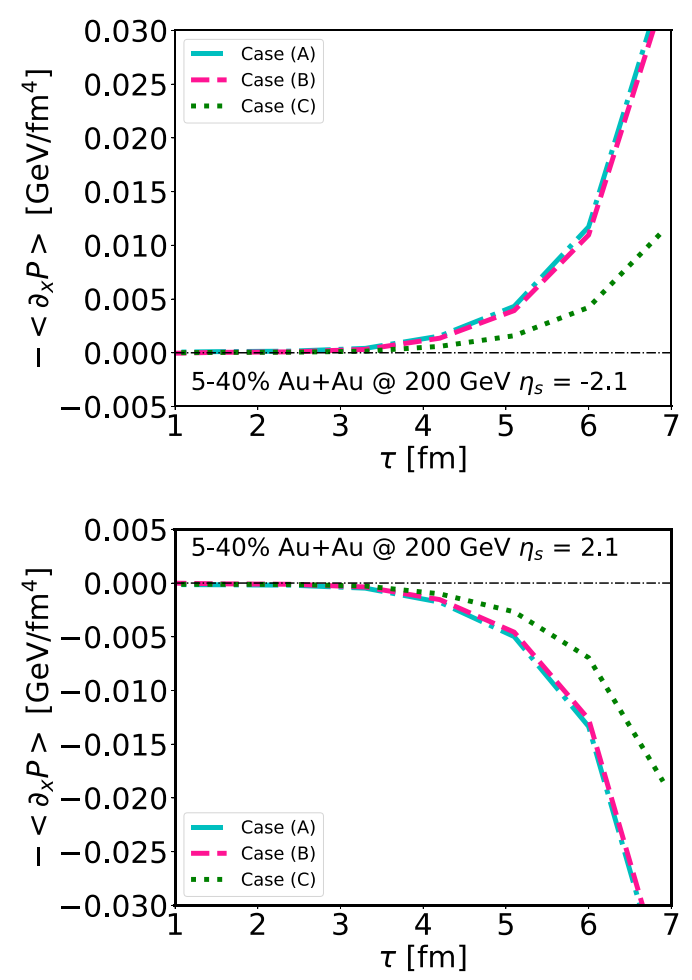

FIG. 5. Time evolution of the average pressure gradient in the $x$ direction at $\eta_{s}=-2.1$ (upper panel) and $\eta_{s}=2.1$ (lower panel) in $5-40 \% \mathrm{Au}+\mathrm{Au}$ collisions at $\sqrt{s_{N N}}=200 \mathrm{GeV}$, compared between three parametrization methods of the initial condition.

where $\gamma=1 / \sqrt{1-v_{x}^{2}-v_{y}^{2}-v_{\eta_{s}}^{2}}$ is the Lorentz boost factor.

Driven by the pressure gradient $-\left\langle\partial_{x} P\right\rangle$ previously shown in Figs. 4 and 5, the average flow velocity $\left\langle v_{x}\right\rangle$ in Fig. 6 is positive (negative) at backward (forward) rapidity for cases (A) and (B). The magnitude of $\left\langle v_{x}\right\rangle$ increases with time as that of the pressure gradient does. A larger $\left|-\left\langle\partial_{x} P\right\rangle\right|$ generates a larger $\left\langle v_{x}\right\rangle$ at $\left|\eta_{s}\right|$ around $\left|\eta_{s}\right| \approx 2$ than around $\left|\eta_{s}\right| \approx 1$. In contrast, $\left\langle v_{x}\right\rangle$ from case (C) shows opposite sign to that from cases (A) and (B) at small $\left|\eta_{s}\right|$, while the same sign beyond $\left|\eta_{s}\right| \approx 2$. This is also consistent with the behaviors of the average pressure gradient at different space-time rapidity from case (C). The average flow velocity of the QGP medium here will directly contribute to the directed flow coefficient of the final-state hadrons emitted from the QGP.

\section{CHARGED PARTICLE YIELD AND DIRECTED FLOW}

With the above setup of energy density initialization and hydrodynamic simulation, we present our results of charged particle yield and directed flow in this section. In particular, we investigate how the directed flow depends on the energy density distribution of the initial state.

To start with, we validate our model setup by comparing to the pseudorapidity distribution of the final-state charged particles in Fig. 7 (for RHIC) and Fig. 8 (for LHC). As discussed earlier, the model parameters summarized in Table II are adjusted to describe these charged particle distributions in the most central collisions at RHIC and LHC. As shown 

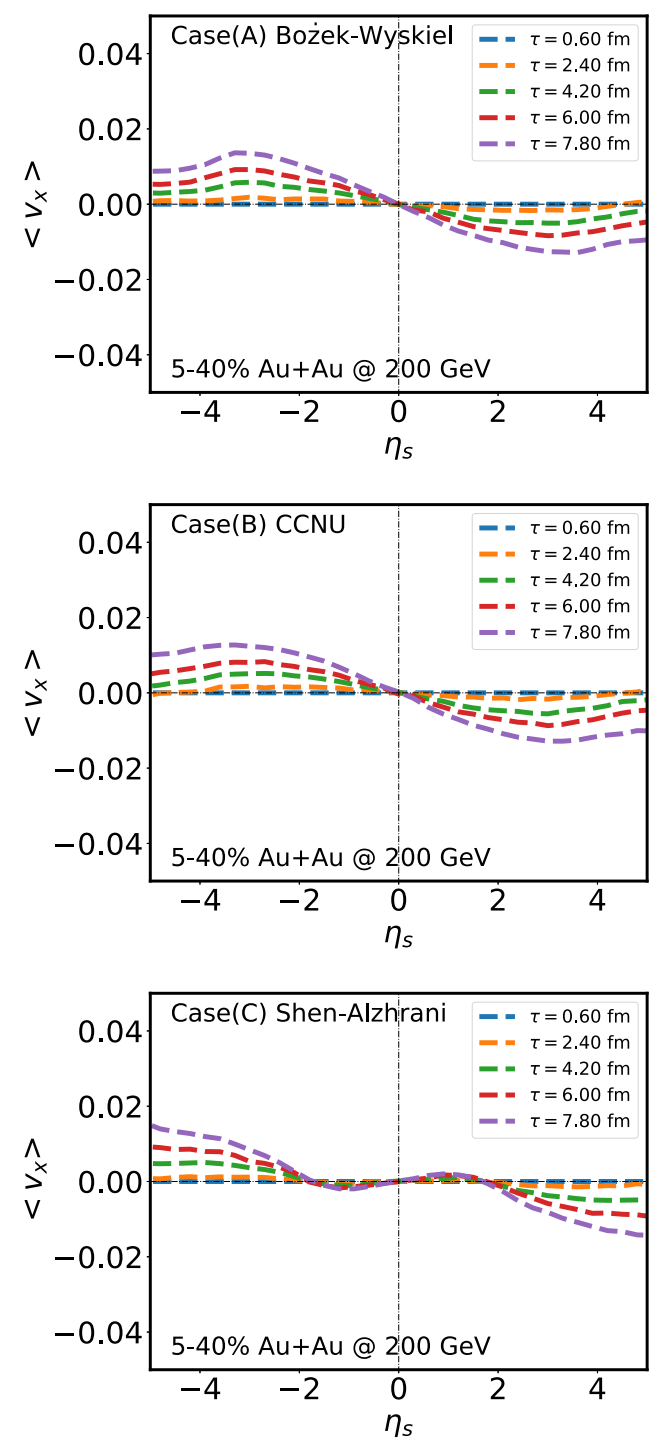

FIG. 6. Space-time rapidity dependence of the average flow velocity in the $x$ direction at different evolution times in 5-40\% $\mathrm{Au}+\mathrm{Au}$ collisions at $\sqrt{s_{N N}}=200 \mathrm{GeV}$, compared between three parametrization methods of the initial condition.

in the figures, after the hydrodynamic parameters are fixed for $0-6 \% \mathrm{Au}+\mathrm{Au}$ collisions at $\sqrt{s_{N N}}=200 \mathrm{GeV}$ and 0 $5 \% \mathrm{~Pb}+\mathrm{Pb}$ collisions at $\sqrt{s_{N N}}=2.76 \mathrm{TeV}$, our calculation provides reasonable descriptions of the PHOBOS data [71] on the $d N_{\mathrm{ch}} / d \eta$ distributions in other centralities at RHIC and the ALICE data [72] at LHC. The same set of parameters in Table II can be applied to the three initial condition models under investigation. This provides a reliable baseline for our further study of the directed flow coefficient.

In the end, we present results on the charged particle directed flow $v_{1}$ as functions of pseudorapidity. Here $v_{1}(\eta)$ is calculated via

$$
v_{1}(\eta)=\left\langle\cos \left(\phi-\Psi_{1}\right)\right\rangle=\frac{\int \cos \left(\phi-\Psi_{1}\right) \frac{d N}{d \eta d \phi} d \phi}{\int \frac{d N}{d \eta d \phi} d \phi},
$$
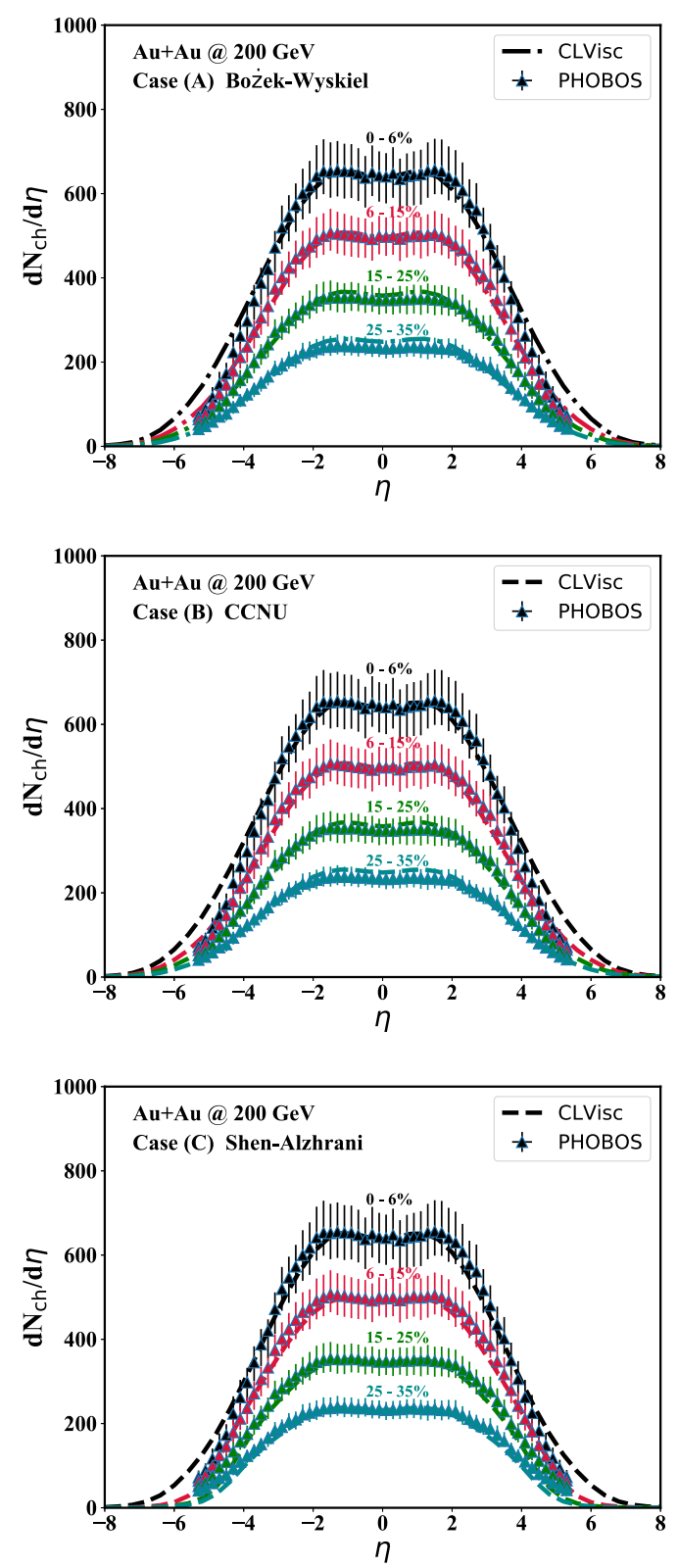

FIG. 7. Pseudorapidity distribution of charged particles in $\mathrm{Au}+$ Au collisions at $\sqrt{s_{N N}}=200 \mathrm{GeV}$ in $0-6 \%, 6-15 \%, 15-25 \%$, and 25-35\% centrality classes, compared between the CLVisc hydrodynamic calculation with three initial condition setups and the PHOBOS data [71].

where $\Psi_{1}$ is the first-order event plane of the collision [44]. Since we use the optical Glauber model (described in Sec. II A) to initialize the energy density distribution of the QGP, event-by-event fluctuations are ignored in this work. As a result, the event plane here should be the same as the spectator plane determined using the deflected neutrons in realistic experimental measurements. A more consistent analysis should be conducted in our future work after event-by-event fluctuations are introduced. The charged particle $v_{1}$ is sensitive to the deformation of the initial energy density, which is governed by $\eta_{m}$ in the 

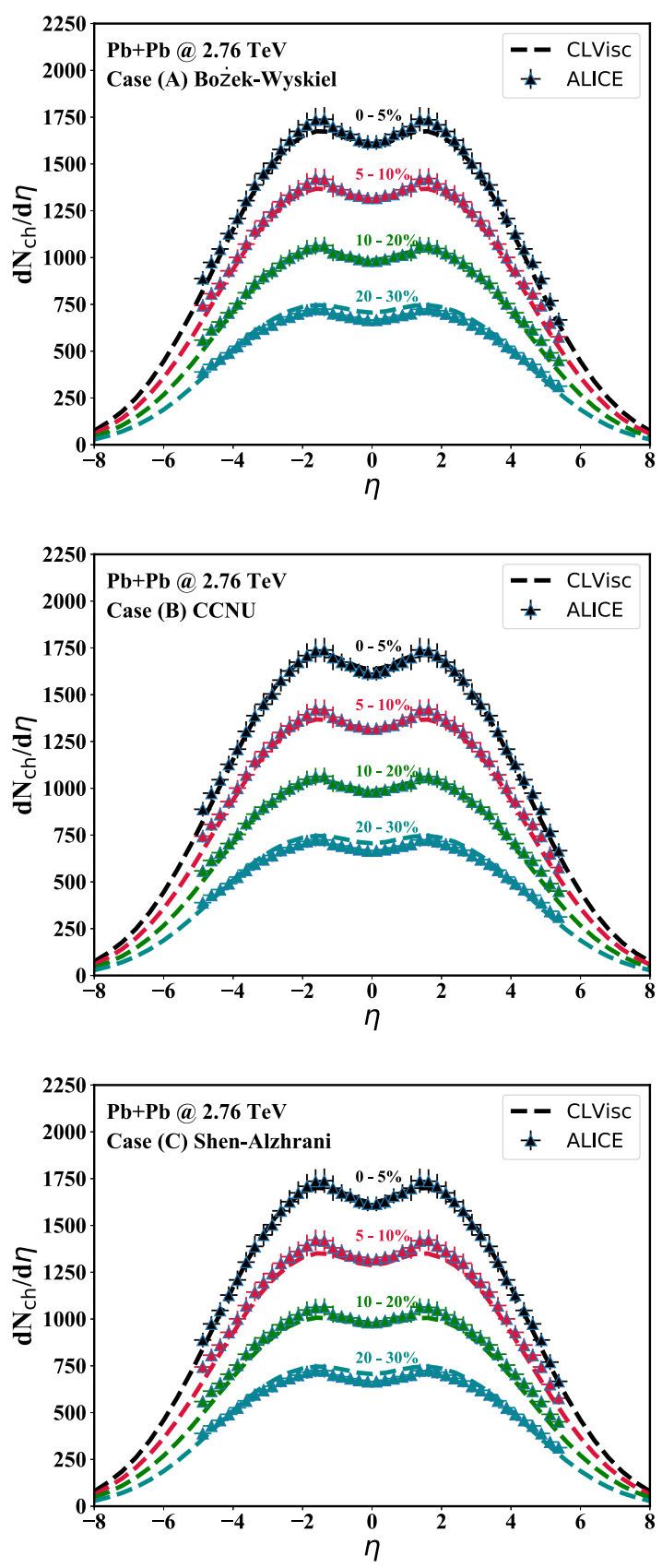

FIG. 8. Pseudorapidity distribution of charged particles in $\mathrm{Pb}+$ $\mathrm{Pb}$ collisions at $\sqrt{s_{N N}}=2.76 \mathrm{TeV}$ in $0-5 \%, 5-10 \%, 10-20 \%$, and 20-30\% centrality classes, compared between the CLVisc hydrodynamic calculation with three initial condition setups and the ALICE data [72].

Bozèk-Wyskiel parametrization, $H_{t}$ in the CCNU parametrization, and $f$ in the Shen-Alzhrani parametrization, as described in Sec. II A.

In case $(\mathrm{A}), \eta_{m}$ determines the forward-backward correlation length [44,73]. A larger value of $\eta_{m}$ in Eq. (5) yields a weaker tilt of the initial energy density distribution and thus a smaller value of charged particle $v_{1}$. In case (B), an alternative way of constructing the tilted initial condition is proposed. A larger value of $H_{t}$ in Eq. (6) results in a more tilted initial
TABLE III. Parameters used in different initial condition models for describing the directed flow coefficient measured at RHIC and LHC. The average impact parameters $(b)$ are used for different centrality bins.

\begin{tabular}{lcccc}
\hline \hline & $\begin{array}{c}200 \mathrm{GeV} \\
\mathrm{Au}+\mathrm{Au} \\
5-40 \%\end{array}$ & $\begin{array}{c}200 \mathrm{GeV} \\
\mathrm{Au}+\mathrm{Au} \\
30-60 \%\end{array}$ & $\begin{array}{c}2.76 \mathrm{TeV} \\
\mathrm{Pb}+\mathrm{Pb} \\
10-20 \%\end{array}$ & $\begin{array}{c}2.76 \mathrm{TeV} \\
\mathrm{Pb}+\mathrm{Pb} \\
30-40 \%\end{array}$ \\
\hline$b(\mathrm{fm})[53,60]$ & 6.69 & 9.70 & 6.50 & 9.40 \\
$\eta_{m}$ & 2.80 & 2.10 & 12.10 & 12.00 \\
$H_{t}$ & 2.90 & 4.30 & 0.70 & 0.70 \\
$f[47]$ & 0.15 & 0.15 & 0.05 & 0.05 \\
\hline \hline
\end{tabular}

energy distribution and therefore a larger magnitude of $v_{1}$ in the end. In case $(\mathrm{C})$, the parameter $f$ varies the longitudinal extent of the fireball and affects the net longitudinal momentum of the hydrodynamic fields [47]. For a given collision system in a given centrality, a larger $f$ leads to a smaller magnitude of $v_{1}$, which is constrained by experimental data. Values of these three parameters used in our calculation are summarized in Table III. These parameters only affect the deformation of the medium geometry, but have very weak impact on the $d N_{\mathrm{ch}} / d \eta$ distributions that have already been fixed by parameters in Table II.

Using these parameters, we show the charged particle $v_{1}$ in $\mathrm{Au}+\mathrm{Au}$ collisions at $\sqrt{s_{N N}}=200 \mathrm{GeV}$ in Fig. 9, upper panel for $5-40 \%$ centrality and lower for $30-60 \%$. We observe parametrizations in our case (A) and (B) result in comparable charged particle $v_{1}$ within $-2<\eta<2$, which is also consistent with the STAR data. As expected, the sign of the charged particle $v_{1}$ is consistent with that of the average flow velocity $v_{x}$ of the QGP fluid-positive (negative) at backward (forward) rapidity. Similar findings for cases (A) and (B) can be observed in Fig. 10 for the charged particle $v_{1}$ in $\mathrm{Pb}+\mathrm{Pb}$ collisions at $\sqrt{s_{N N}}=2.76 \mathrm{TeV}$, upper panel for $10-20 \%$ and lower for 30-40\%.

For case (C), within our current hydrodynamic framework, we are unable to describe the charged particle $v_{1}$ at either RHIC or LHC by adjusting its $f$ parameter. In the present study, we use $f=0.15$ at RHIC and $f=0.05$ at LHC as suggested by the $\Lambda$ polarization calculation [47,74]. If one increases the value of $f$, the slope of $v_{1}$ vs $\eta$ will increase near the midpseudorapidity region and further deviate from the experimental data. This implies the importance of the tilted deformation of the initial energy density distribution in understanding the $\eta$ dependence of $v_{1}$ observed in experiments. Our results for case $(\mathrm{C})$ here are qualitatively consistent with those of previous studies $[44,46]$. However, as suggested by Refs. [46,47], introducing net baryon current into hydrodynamic simulation, varying the $\eta_{w}$ and $\sigma_{\eta}$ parameters in Eq. (14), or taking into account hadronic rescatterings after the QGP phase may further affect the charged particle $v_{1}$, or even flip its sign in the mid- $\eta$ region. These effects are not the focus of our present study on the medium geometry effects and will be left for a future investigation.

In the present study, the directed flow coefficients above are analyzed with soft hadrons within $0<p_{\mathrm{T}}<3.5 \mathrm{GeV}$. Since 

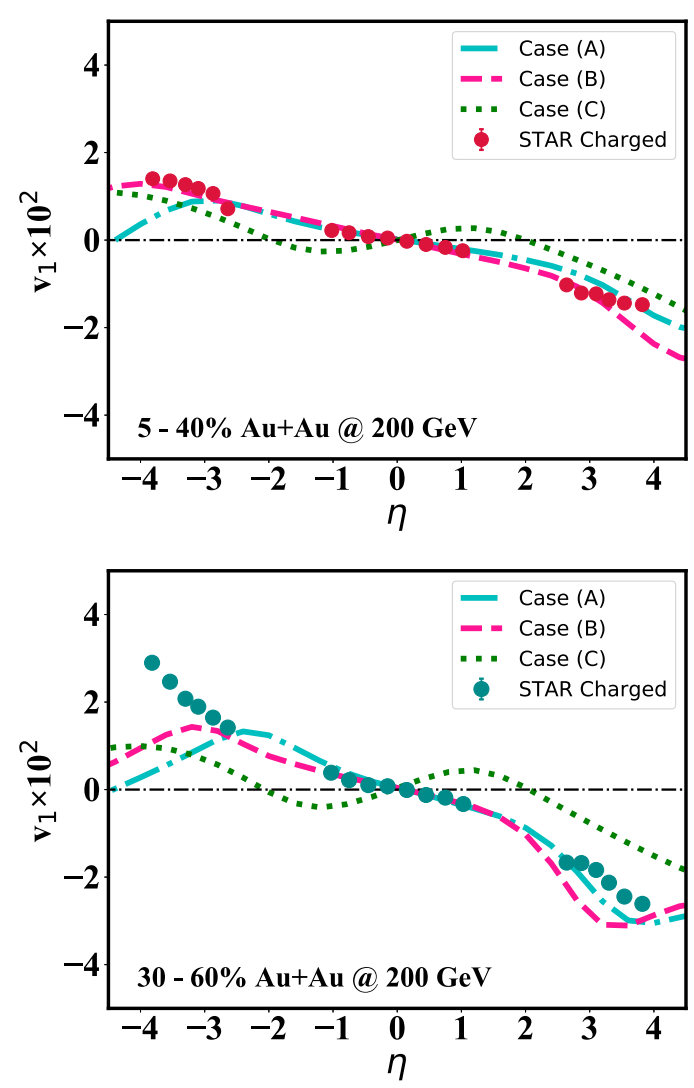

FIG. 9. Pseudorapidity dependence of the directed flow coefficient in 5-40\% (upper panel) and 30-60\% (lower panel) $\mathrm{Au}+\mathrm{Au}$ collisions at $\sqrt{s_{N N}}=200 \mathrm{GeV}$, compared between the CLVisc hydrodynamic calculation with three initial condition setups and the STAR data [32,55].

we use the smooth initial condition from the optical Glauber model, our discussion is restricted to the rapidity-odd component of $v_{1}$ here. The rapidity-even component, including its nontrivial $p_{\mathrm{T}}$ dependence even at midrapidity [75-77], is beyond the scope of this work.

\section{SUMMARY AND OUTLOOK}

In this work, we have performed a systematic study on how the geometry of the initial energy density profile affects the charged particle directed flow in high-energy nuclear collisions. Three different parametrization setupscase (A) Bozèk-Wyskiel, case (B) CCNU, and case (C) Shen-Alzhrani-are compared for the initial energy density distribution, and their subsequent time evolutions are simulated using the $(3+1)-\mathrm{D}$ viscous hydrodynamic model CLVisc.

Within this framework, we have found the counterclockwise tilt of the initial energy density profile in the $x-\eta_{s}$ plane, as generated by our cases (A) and (B), yields an increasing (decreasing) average pressure gradient $-\left\langle\partial_{x} P\right\rangle$ from zero with respect to time at backward (forward) rapidity. The magnitude of this pressure gradient appears larger at larger $\left|\eta_{s}\right|$. This further leads to a negative slope of the average QGP flow velocity $\left\langle v_{x}\right\rangle$ with respect to the space-time rapidity within
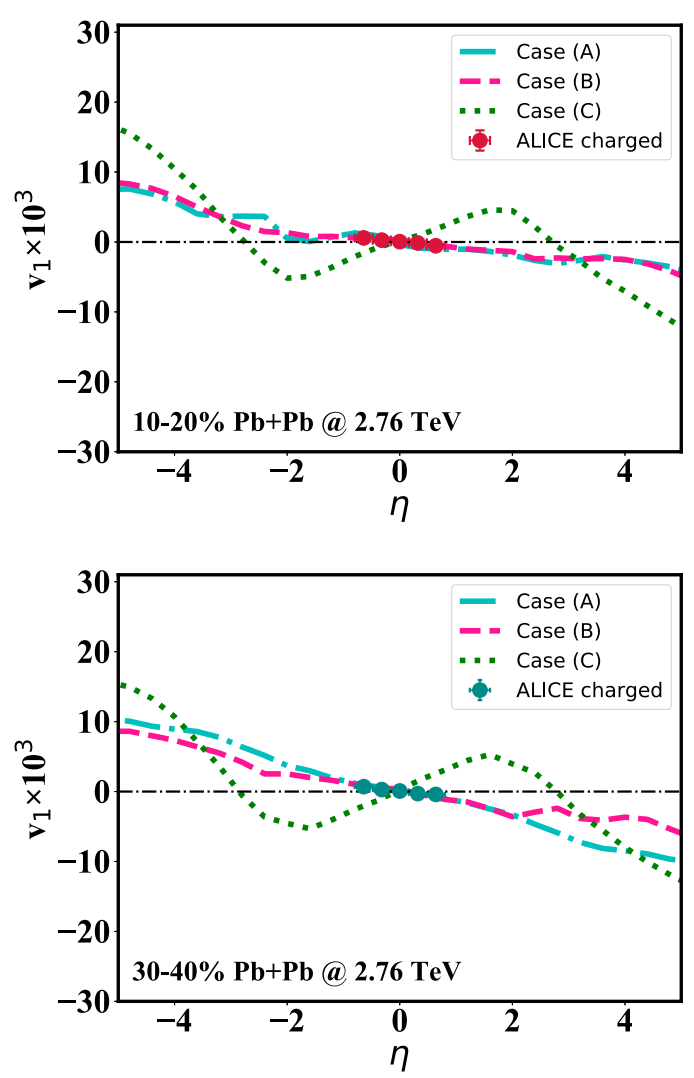

FIG. 10. Pseudorapidity dependence of the directed flow coefficient in 10-20\% (upper panel) and 30-40\% (lower panel) $\mathrm{Pb}+\mathrm{Pb}$ collisions at $\sqrt{s_{N N}}=2.76 \mathrm{TeV}$, compared between the CLVisc hydrodynamic calculation with three initial condition setups and the ALICE data [56].

$\left|\eta_{s}\right|<2$, and in the end the same behavior of $v_{1}$ versus $\eta$ of the final-state charged particles emitted from the QGP. In contrast, without the tilted deformation in the central space-time rapidity region, the shifted initial geometry along the longitudinal direction alone in case (C) results in the opposite sign of the $x$ component of the average pressure gradient, the average QGP flow velocity, and the charged particle $v_{1}$ at mid- $\eta_{s}$ (or $\eta$ ). At large space-time rapidity, the stronger deformation from case (C) can also generate similar time and $\eta_{s}(\eta)$ dependence of the above quantities as cases (A) and (B). A comparison to the RHIC and LHC data indicates the essential role of the tilted initial energy density profile in helping understand the observed charged particle $v_{1}$. We note that the correct sign of $-\left\langle\partial_{x} P\right\rangle$ at backward or forward rapidity is the key that drives the final directed flow of soft hadrons. This is more important than the detailed form of how to parametrize the initial condition. Alternative parametrization methods other than the tilted profile may exist, which lead to the similar $\eta_{s}$ dependence of the pressure gradient.

Our study constitutes a step forward in revealing the source of the directed flow generated in relativistic heavy-ion collisions. Nevertheless, apart from the titled deformation of the initial geometry, other sources exist for the development of directed flow. For instance, (1) deviations from the Bjorken flow in the initial condition could provide additional 
contribution to the directed flow [47]. In particular, they could affect the flow pattern of baryons and thus the bulk medium profile [44]. (2) The strong electromagnetic field produced in the early stage of noncentral heavy-ion collisions results in deflection of charged particles and influence the charged particle $v_{1}[73,78,79]$, although this effect is suggested small compared to the titled initial geometry [73]. (3) The charged particle $v_{1}$ can also be contributed by the initial decelerated baryons and hadronic cascade after the QGP phase, especially at lower collision energy [46,47]. Moreover, a combination of hydrodynamic model and afterburner hadronic transport, such as UrQMD [68,80] and SMASH $[69,81]$, are found necessary for more stringent constraints on the initial stage of nuclear collisions. These should be incorporated in our future calculation for a more precise understanding of the directed flow.

\section{ACKNOWLEDGMENTS}

We are grateful for helpful discussions with Chun Shen, Jiaxing Zhao, Guang-You Qin, and Long-Gang Pang. This work was supported by the National Natural Science Foundation of China (NSFC) under Grants No. 11935007, No. 12175122, and No. 2021-867; Guangdong Major Project of Basic and Applied Basic Research No. 2020B0301030008; the Natural Science Foundation of Hubei Province No. 2021CFB272; the Education Department of Hubei Province of China with Young Talents Project No. Q20212703; and the Xiaogan Natural Science Foundation under Grant No. XGKJ2021010016. Computational resources were provided by the Center of Scientific Computing at the Department of Physics and Electronic-Information Engineering, Hubei Engineering University.
[1] S. S. Adler et al., Elliptic Flow of Identified Hadrons in $\mathrm{Au}+\mathrm{Au}$ Collisions at $s(N N)^{* *}(1 / 2)=200 \mathrm{GeV}$, Phys. Rev. Lett. 91, 182301 (2003).

[2] K. Aamodt et al., Elliptic Flow of Charged Particles in $\mathrm{Pb}-\mathrm{Pb}$ Collisions at 2.76 TeV, Phys. Rev. Lett. 105, 252302 (2010).

[3] S. Chatrchyan et al., Measurement of the elliptic anisotropy of charged particles produced in $\mathrm{Pb}-\mathrm{Pb}$ collisions at $\sqrt{s_{N N}}=$ 2.76 TeV, Phys. Rev. C 87, 014902 (2013).

[4] J.-Y. Ollitrault, Anisotropy as a signature of transverse collective flow, Phys. Rev. D 46, 229 (1992).

[5] D. H. Rischke, S. Bernard, and J. A. Maruhn, Relativistic hydrodynamics for heavy ion collisions. 1. General aspects and expansion into vacuum, Nucl. Phys. A 595, 346 (1995).

[6] H. Sorge, Elliptical Flow: A Signature for Early Pressure in Ultrarelativistic Nucleus-Nucleus Collisions, Phys. Rev. Lett. 78, 2309 (1997).

[7] S. A. Bass, M. Gyulassy, H. Stoecker, and W. Greiner, Signatures of quark gluon plasma formation in high-energy heavy ion collisions: A critical review, J. Phys. G 25, R1 (1999).

[8] C. E. Aguiar, Y. Hama, T. Kodama, and T. Osada, Event-byevent fluctuations in hydrodynamical description of heavy ion collisions, Nucl. Phys. A 698, 639 (2002).

[9] E. Shuryak, Why does the quark gluon plasma at RHIC behave as a nearly ideal fluid? Prog. Part. Nucl. Phys. 53, 273 (2004).

[10] M. Gyulassy and L. McLerran, New forms of QCD matter discovered at RHIC, Nucl. Phys. A 750, 30 (2005).

[11] W. Broniowski, P. Bozek, and M. Rybczynski, Fluctuating initial conditions in heavy-ion collisions from the Glauber approach, Phys. Rev. C 76, 054905 (2007).

[12] R. P. G. Andrade, F. Grassi, Y. Hama, T. Kodama, and W. L. Qian, Importance of Granular Structure in the Initial Conditions for the Elliptic Flow, Phys. Rev. Lett. 101, 112301 (2008).

[13] T. Hirano and Y. Nara, Eccentricity fluctuation effects on elliptic flow in relativistic heavy ion collisions, Phys. Rev. C 79, 064904 (2009).

[14] B. Schenke, S. Jeon, and C. Gale, Elliptic and Triangular Flow in Event-by-Event (3+1)D Viscous Hydrodynamics, Phys. Rev. Lett. 106, 042301 (2011).

[15] Z. Qiu and U. W. Heinz, Event-by-event shape and flow fluctuations of relativistic heavy-ion collision fireballs, Phys. Rev. C 84, 024911 (2011).
[16] U. Heinz and R. Snellings, Collective flow and viscosity in relativistic heavy-ion collisions, Ann. Rev. Nucl. Part. Sci. 63, 123 (2013).

[17] P. Huovinen, Hydrodynamics at RHIC and LHC: What have we learned? Int. J. Mod. Phys. E 22, 1330029 (2013).

[18] C. Gale, S. Jeon, and B. Schenke, Hydrodynamic modeling of heavy-ion collisions, Int. J. Mod. Phys. A 28, 1340011 (2013).

[19] P. Bozek and W. Broniowski, Collective dynamics in highenergy proton-nucleus collisions, Phys. Rev. C 88, 014903 (2013).

[20] G.-Y. Qin and B. Müller, Elliptic and triangular flow anisotropy in deuteron-gold collisions at $\sqrt{s_{N N}}=200 \mathrm{GeV}$ at RHIC and in proton-lead collisions at $\sqrt{s_{N N}}=5.02 \mathrm{TeV}$ at the LHC, Phys. Rev. C 89, 044902 (2014).

[21] K. Dusling, W. Li, and B. Schenke, Novel collective phenomena in high-energy proton-proton and proton-nucleus collisions, Int. J. Mod. Phys. E 25, 1630002 (2016).

[22] P. Romatschke and U. Romatschke, Relativistic Fluid Dynamics in and out of Equilibrium, Cambridge Monographs on Mathematical Physics (Cambridge University Press, Cambridge, UK, 2019).

[23] R. D. Weller and P. Romatschke, One fluid to rule them all: Viscous hydrodynamic description of event-by-event central $p+p, p+\mathrm{Pb}$, and $\mathrm{Pb}+\mathrm{Pb}$ collisions at $\sqrt{s}=5.02 \mathrm{TeV}$, Phys. Lett. B 774, 351 (2017).

[24] W. Zhao, C. M. Ko, Y.-X. Liu, G.-Y. Qin, and H. Song, Probing the Partonic Degrees of Freedom in High-Multiplicity $p$-Pb Collisions at $\sqrt{s_{N N}}=5.02 \mathrm{TeV}$, Phys. Rev. Lett. 125, 072301 (2020).

[25] H. Song, S. A. Bass, U. Heinz, T. Hirano, and C. Shen, $200 \mathrm{~A} \mathrm{GeV} \mathrm{Au+Au} \mathrm{Collisions} \mathrm{Serve} \mathrm{a} \mathrm{Nearly} \mathrm{Perfect} \mathrm{Quark-}$ Gluon Liquid, Phys. Rev. Lett. 106, 192301 (2011); Erratum: 200 A GeV Au + Au Collisions Serve a Nearly Perfect QuarkGluon Liquid [Phys. Rev. Lett. 106, 192301 (2011)], 109, 139904(E) (2012).

[26] J. E. Bernhard, J. S. Moreland, and S. A. Bass, Bayesian estimation of the specific shear and bulk viscosity of quark-gluon plasma, Nat. Phys. 15, 1113 (2019).

[27] S. Voloshin and Y. Zhang, Flow study in relativistic nuclear collisions by Fourier expansion of azimuthal particle distributions, Z. Phys. C 70, 665 (1996). 
[28] A. Bilandzic, R. Snellings, and S. Voloshin, Flow analysis with cumulants: Direct calculations, Phys. Rev. C 83, 044913 (2011).

[29] M. Gyulassy, K. A. Frankel, and H. Stoecker, Do nuclei flow at high energies? Phys. Lett. B 110, 185 (1982).

[30] H. A. Gustafsson et al., Collective Flow Observed in Relativistic Nuclear Collisions, Phys. Rev. Lett. 52, 1590 (1984).

[31] M. A. Lisa, U. W. Heinz, and U. A. Wiedemann, Tilted pion sources from azimuthally sensitive HBT interferometry, Phys. Lett. B 489, 287 (2000).

[32] J. Adams et al., Azimuthal anisotropy in Au+Au collisions at $s(N N) * *(1 / 2)=200-\mathrm{GeV}$, Phys. Rev. C 72, 014904 (2005).

[33] L. Adamczyk et al., Beam-Energy Dependence of the Directed Flow of Protons, Antiprotons, and Pions in $\mathrm{Au}+\mathrm{Au}$ Collisions, Phys. Rev. Lett. 112, 162301 (2014).

[34] L. Adamczyk et al., Beam-Energy Dependence of Directed Flow of $\Lambda, \bar{\Lambda}, K^{ \pm}, K_{s}^{0}$ and $\phi$ in Au+Au Collisions, Phys. Rev. Lett. 120, 062301 (2018).

[35] J. Adam et al., First Observation of the Directed Flow of $D^{0}$ and $\overline{D^{0}}$ in Au+Au Collisions at $\sqrt{s_{N N}}=200 \mathrm{GeV}$, Phys. Rev. Lett. 123, 162301 (2019).

[36] S. Acharya et al., Probing the Effects of Strong Electromagnetic Fields with Charge-Dependent Directed Flow in $\mathrm{Pb}-\mathrm{Pb}$ Collisions at the LHC, Phys. Rev. Lett. 125, 022301 (2020).

[37] J. Adam et al., Bulk properties of the system formed in $\mathrm{Au}+\mathrm{Au}$ collisions at $\sqrt{s_{N N}}=14.5 \mathrm{GeV}$ at the BNL STAR detector, Phys. Rev. C 101, 024905 (2020).

[38] S. Singha, P. Shanmuganathan, and D. Keane, The first moment of azimuthal anisotropy in nuclear collisions from AGS to LHC energies, Adv. High Energy Phys. 2016, 2836989 (2016).

[39] Y. Nara, H. Niemi, A. Ohnishi, and H. Stöcker, Examination of directed flow as a signature of the softest point of the equation of state in QCD matter, Phys. Rev. C 94, 034906 (2016).

[40] S. Chatterjee and P. Bożek, Large Directed Flow of Open Charm Mesons Probes the Three Dimensional Distribution of Matter in Heavy Ion Collisions, Phys. Rev. Lett. 120, 192301 (2018).

[41] C. Zhang, J. Chen, X. Luo, F. Liu, and Y. Nara, Beam energy dependence of the squeeze-out effect on the directed and elliptic flow in $\mathrm{Au}+\mathrm{Au}$ collisions in the high baryon density region, Phys. Rev. C 97, 064913 (2018).

[42] C.-Q. Guo, C.-J. Zhang, and J. Xu, Revisiting directed flow in relativistic heavy-ion collisions from a multiphase transport model, Eur. Phys. J. A 53, 233 (2017).

[43] A. Adil and M. Gyulassy, 3D jet tomography of twisted strongly coupled quark gluon plasmas, Phys. Rev. C 72, 034907 (2005).

[44] P. Bozek and I. Wyskiel, Directed flow in ultrarelativistic heavyion collisions, Phys. Rev. C 81, 054902 (2010).

[45] B. Chen, M. Hu, H. Zhang, and J. Zhao, Probe the tilted quarkgluon plasma with charmonium directed flow, Phys. Lett. B 802, 135271 (2020).

[46] C. Shen and S. Alzhrani, Collision-geometry-based 3D initial condition for relativistic heavy-ion collisions, Phys. Rev. C 102, 014909 (2020).

[47] S. Ryu, V. Jupic, and C. Shen, Probing early-time longitudinal dynamics with the $\Lambda$ hyperon's spin polarization in relativistic heavy-ion collisions, Phys. Rev. C 104, 054908 (2021).

[48] S. Chatterjee and P. Bozek, Interplay of drag by hot matter and electromagnetic force on the directed flow of heavy quarks, Phys. Lett. B 798, 134955 (2019).

[49] A. Beraudo, A. De Pace, M. Monteno, M. Nardi, and F. Prino, Rapidity dependence of heavy-flavour production in heavy-ion collisions within a full $3+1$ transport approach: Quenching, elliptic and directed flow, JHEP 05 (2021) 279.

[50] L. Oliva, S. Plumari, and V. Greco, Directed flow of D mesons at RHIC and LHC: Non-perturbative dynamics, longitudinal bulk matter asymmetry, and electromagnetic fields, JHEP 05 (2021) 034.

[51] Z.-F. Jiang, C. B. Yang, and Q. Peng, Directed flow of charged particles within idealized viscous hydrodynamics at energies available at the BNL Relativistic Heavy Ion Collider and at the CERN Large Hadron Collider, Phys. Rev. C 104, 064903 (2021).

[52] L.-G. Pang, H. Petersen, Q. Wang, and X.-N. Wang, Vortical Fluid and $\Lambda$ Spin Correlations in High-Energy Heavy-Ion Collisions, Phys. Rev. Lett. 117, 192301 (2016).

[53] L.-G. Pang, H. Petersen, and X.-N. Wang, Pseudorapidity distribution and decorrelation of anisotropic flow within the open-computing-language implementation CLVisc hydrodynamics, Phys. Rev. C 97, 064918 (2018).

[54] X.-Y. Wu, L.-G. Pang, G.-Y. Qin, and X.-N. Wang, Longitudinal fluctuations and decorrelations of anisotropic flows at energies available at the CERN Large Hadron Collider and at the BNL Relativistic Heavy Ion Collider, Phys. Rev. C 98, 024913 (2018).

[55] B. I. Abelev et al., System-Size Independence of Directed Flow at the Relativistic Heavy-Ion Collider, Phys. Rev. Lett. 101, 252301 (2008).

[56] B. Abelev et al., Directed Flow of Charged Particles at Midrapidity Relative to the Spectator Plane in $\mathrm{Pb}-\mathrm{Pb}$ Collisions at $\sqrt{s_{N N}}=2.76 \mathrm{TeV}$, Phys. Rev. Lett. 111, 232302 (2013).

[57] P. Bozek, Flow and interferometry in $3+1$ dimensional viscous hydrodynamics, Phys. Rev. C 85, 034901 (2012).

[58] C. Loizides, J. Kamin, and D. d'Enterria, Improved Monte Carlo Glauber predictions at present and future nuclear colliders, Phys. Rev. C 97, 054910 (2018); Erratum: Improved Monte Carlo Glauber predictions at present and future nuclear colliders [Phys. Rev. C 97, 054910 (2018)], 99, 019901(E) (2019).

[59] T. Hirano and K. Tsuda, Collective flow and two pion correlations from a relativistic hydrodynamic model with early chemical freezeout, Phys. Rev. C 66, 054905 (2002).

[60] T. Hirano, U. W. Heinz, D. Kharzeev, R. Lacey, and Y. Nara, Hadronic dissipative effects on elliptic flow in ultrarelativistic heavy-ion collisions, Phys. Lett. B 636, 299 (2006).

[61] W. Chen, S. Cao, T. Luo, L.-G. Pang, and X.-N. Wang, Effects of jet-induced medium excitation in $\gamma$-hadron correlation in $A+A$ collisions, Phys. Lett. B 777, 86 (2018).

[62] Y. He, L.-G. Pang, and X.-N. Wang, Bayesian Extraction of Jet Energy Loss Distributions in Heavy-Ion Collisions, Phys. Rev. Lett. 122, 252302 (2019).

[63] Z. F. Jiang, D. She, C. B. Yang, and D. Hou, Perturbation solutions of relativistic viscous hydrodynamics forlongitudinally expanding fireballs, Chin. Phys. C 44, 084107 (2020).

[64] Z. F. Jiang, C. B. Yang, C. Ding, and X.-Y. Wu, Pseudo-rapidity distribution from a perturbative solution of viscous hydrodynamics for heavy ion collisions at RHIC and LHC, Chin. Phys. C 42, 123103 (2018).

[65] G. S. Denicol, H. Niemi, E. Molnar, and D. H. Rischke, Derivation of transient relativistic fluid dynamics from the Boltzmann equation, Phys. Rev. D 85, 114047 (2012); Erratum: Derivation of transient relativistic fluid dynamics from the Boltzmann equation [Phys. Rev. D 85, 114047 (2012)], 91, 039902(E) (2015). 
[66] P. Romatschke, New developments in relativistic viscous hydrodynamics, Int. J. Mod. Phys. E 19, 1 (2010).

[67] S. Borsanyi, Z. Fodor, C. Hoelbling, S. D. Katz, S. Krieg, and K. K. Szabo, Full result for the QCD equation of state with $2+1$ flavors, Phys. Lett. B 730, 99 (2014).

[68] W. Zhao, W. Ke, W. Chen, T. Luo, and X.-N. Wang, From Hydrodynamics to Jet Quenching, Coalescence, and Hadron Cascade: A Coupled Approach to Solving the RAA $\otimes$ v2 puzzle, Phys. Rev. Lett. 128, 022302 (2022).

[69] X.-Y. Wu, G.-Y. Qin, L.-G. Pang, and X.-N. Wang, (3+1)-D viscous hydrodynamics CLVisc at finite net baryon density: Identified particle spectra, anisotropic flows, and flow fluctuations across BES energies, arXiv:2107.04949.

[70] F. Cooper and G. Frye, Comment on the single particle distribution in the hydrodynamic and statistical thermodynamic models of multiparticle production, Phys. Rev. D 10, 186 (1974).

[71] B. Alver et al., Phobos results on charged particle multiplicity and pseudorapidity distributions in $\mathrm{Au}+\mathrm{Au}, \mathrm{Cu}+\mathrm{Cu}, d+\mathrm{Au}$, and $p+p$ collisions at ultra-relativistic energies, Phys. Rev. C 83, 024913 (2011).

[72] J. Adam et al., Centrality evolution of the charged-particle pseudorapidity density over a broad pseudorapidity range in $\mathrm{Pb}-\mathrm{Pb}$ collisions at $\sqrt{s_{N N}}=2.76 \mathrm{TeV}$, Phys. Lett. B 754, 373 (2016).

[73] G. Inghirami, M. Mace, Y. Hirono, L. Del Zanna, D. E. Kharzeev, and M. Bleicher, Magnetic fields in heavy ion collisions: flow and charge transport, Eur. Phys. J. C 80, 293 (2020).

[74] C. Shen (private communications).

[75] D. Teaney and L. Yan, Triangularity and dipole asymmetry in heavy ion collisions, Phys. Rev. C 83, 064904 (2011).

[76] M. Luzum and J.-Y. Ollitrault, Directed Flow at Midrapidity in Heavy-Ion Collisions, Phys. Rev. Lett. 106, 102301 (2011).

[77] C. Gale, S. Jeon, B. Schenke, P. Tribedy, and R. Venugopalan, Event-by-Event Anisotropic Flow in Heavy-Ion Collisions from Combined Yang-Mills and Viscous Fluid Dynamics, Phys. Rev. Lett. 110, 012302 (2013).

[78] U. Gursoy, D. E. Kharzeev, and K. Rajagopal, Magnetohydrodynamics, charged currents, and directed flow in heavy ion collisions, Phys. Rev. C 89, 054905 (2014).

[79] U. Gürsoy, D. E. Kharzeev, E. Marcus, K. Rajagopal, and C. Shen, Charge-dependent flow induced by magnetic and electric fields in heavy ion collisions, Phys. Rev. C 98, 055201 (2018).

[80] S. A. Bass et al., Microscopic models for ultrarelativistic heavy ion collisions, Prog. Part. Nucl. Phys. 41, 255 (1998).

[81] H. Petersen, D. Oliinychenko, M. Mayer, J. Staudenmaier, and S. Ryu, SMASH-A new hadronic transport approach, Nucl. Phys. A 982, 399 (2019). 